\title{
Result Analysis of Smoke Detection in Video for Early Warnings using Static and Dynamic Features
}

\author{
Ashish A. Narwade \\ Research Scholar \\ Government College of Engineering Aurangabad
}

\author{
Vrishali A. Chakkarwar \\ Asst. Professor, \\ Government College of Engineering, Aurangabad
}

\begin{abstract}
This paper presents result analysis of proposed method of smoke detection in video based on image processing to provide an early warning of anomalous events. The experimental results show that an adaptive background subtraction method, HSV color model and SVM classifier provides more accurate results in the segmentation and detection of smoke region in video. Also it shows that smoke features helps in decision making that is whether the segmented region in video is of smoke or not, hence this improves the reliability of system. The proposed method reduces the false detection and increases the smoke detection rate.

This paper is organized into following sections. Section 1 contains introduction to smoke detection. Section 2 contains description of the proposed method. Section 3 contains result analysis of the proposed method. Section 4 contains the conclusion of the work.
\end{abstract}

\section{General Terms}

Smoke detection in video.

\section{Keywords}

Adaptive Background Subtraction, HSV color space, SVM classifier.

\section{INTRODUCTION}

Smoke detection in video systems are one of the most important components in video surveillance systems used to monitor anomalous events such as fire in factories, shopping malls, railways, houses, buses, offices, submarines, environment or cigarette smoking in public areas, etc. as part of an early warning mechanism that reports preferably the start of fire or other hazard.

An image processing approach to smoke detection in video improves conventional passive detection system through automated smoke region detection and segmentation of smoke region. Smoke detection have initiated a wide variety of applications in access control, damage control, hence helps to mitigate effects of fire and smoke.

So, the aim is to detect the smoke using smoke features, as early as possible [1-3] to give more accurate and fast response. Early detection is essential in order to provide an effective response to emergencies and damages and also to provide appropriate actions to mitigate the effects of the smoke and fire can be taken. These actions generally include the evacuation of occupants from danger zone to a safe place and the initiation of extinguishment activities either automatic or manual [3].

In the proposed methodology image processing approach to smoke detection is used [5] [7]. In the initial phase of detection process motion and color features of smoke are used to separate or differentiate stationary, non-stationary smoke region and region with smoke color. For this purpose adaptive background subtraction and HSV color model is used. In the decision or classification step smoke area and other features are used, these features are used to train SVM classifier for classifying segmented region as smoke or non smoke.

\section{SMOKE DETECTION METHOD}

The propose method has four phases for detecting presence of smoke in video: In first phase, all the moving region in video are detected using adaptive background subtraction method; In second phase, detecting objects that match with smoke color from moving object in video; In third step, area and perimeter disorder of segmented smoke regions are calculated; In fourth step, SVM classifier is trained with calculated smoke features to classify the detected region as smoke or non-smoke with warning message on screen.

The flow of proposed method for smoke detection in video is shown Fig 1.

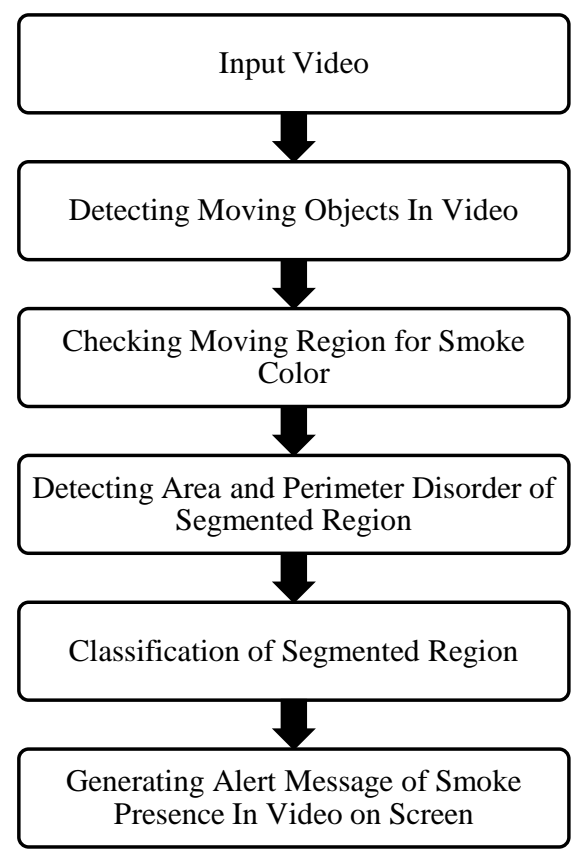

Fig 1: Flow chart of smoke detection in video

Initially, a video is given as input to the system from video dataset, then frames are obtained from input video and further processing is done on frames. To reduce or minimize noise, etc. from video some sort of preprocessing is also performed. The main steps of proposed methods are: 


\subsection{Detecting moving objects in a video using adaptive background subtraction method}

As, smoke detection system can be used for indoor as well as in outdoor environment videos, the background model need to adapt itself to the dynamic changes such as global illumination, etc. Hence, here adaptive background subtraction method is used [3], in which reference background and threshold is dynamically updated with incoming frames. According to adaptive background subtraction method, first, background frame is set as:

Bframe $=\left\{\begin{array}{l}\text { Bframe } \\ \text { when, current frame is first frame } \\ (((1-u) * \text { thisFrame })+(u * \text { Bframe }))\end{array}\right.$

\section{Otherwise}

Where, Bframe is background frame, thisFrame is current frame, and $\mathrm{u}$ is real and positive constant close to one.

After this, difference frame for detecting moving objects in frame using background (Bframe) frame and current frame (thisFrame) calculated, as:

$$
\text { dif }=(\text { thisFrame }-(\text { Bframe })) ;
$$

Then, adaptive threshold is applied to difference image obtained from current and background frame, as:

$$
\text { Th }=\left\{\begin{array}{l}
1 \\
\text { when, current frame is first frame } \\
((u * 0.1)+((1-u) *(2 * t h l))) ;
\end{array}\right.
$$

Otherwise

Where, Th is threshold, $\mathrm{u}$ is real positive constant and thl is threshold level obtained from graythresh method.

Finally using Bframe and Th difference frame is obtained as:

$$
\text { dif }=i m 2 b w(\text { grayImage, } T h) ;
$$

Where, dif is difference frame, grayImage is obtained from rgb image of dif and Th is the threshold

\subsection{Detecting objects that match with smoke color using HSV color model}

As, smoke has static feature of color i.e. gray color it can be used to separate it from non-smoke moving object which is the output of the previous step [2]. In this step, difference frames in RGB color space are converted into HSV color space using standard RGB to HSV.

$$
\text { hsvImage }=\text { rgb2hsv (dif); }
$$

The values of the three components $\mathrm{H}, \mathrm{S}$, and $\mathrm{V}$ are computed and then, setting low and high threshold to $\mathrm{H}, \mathrm{S}$, and $\mathrm{V}$ images band respectively obtain the segmented region having same color as color of smoke. In this step region having the smoke color is find out which is consider as a candidate region for further processing.

\subsection{Calculating area and perimeter disorder of segmented region}

In the classification process dynamic features of smoke like area and perimeter disorder of segmented smoke region are used for differentiating between smoke and non smoke region from detected region in previous steps [2][6]. As an example of this step results are shown in Fig 2:

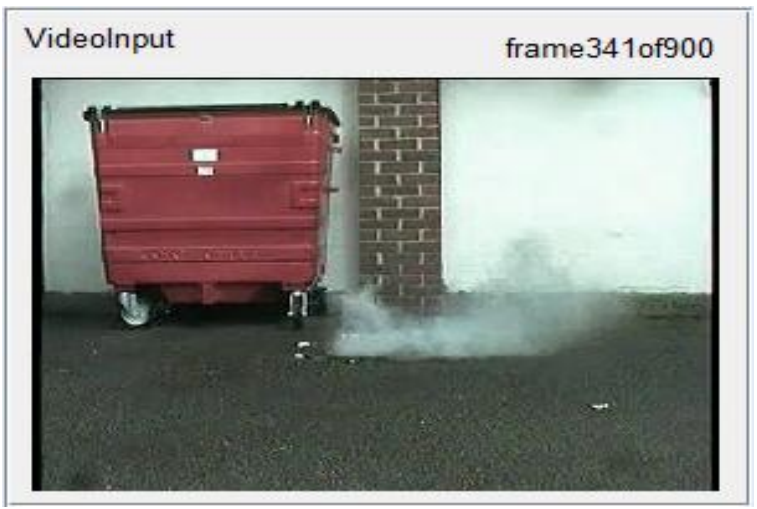

(a)

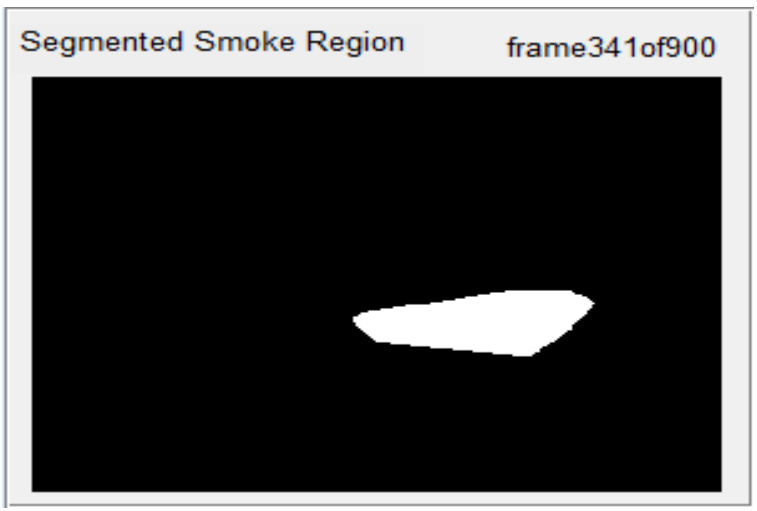

(b)

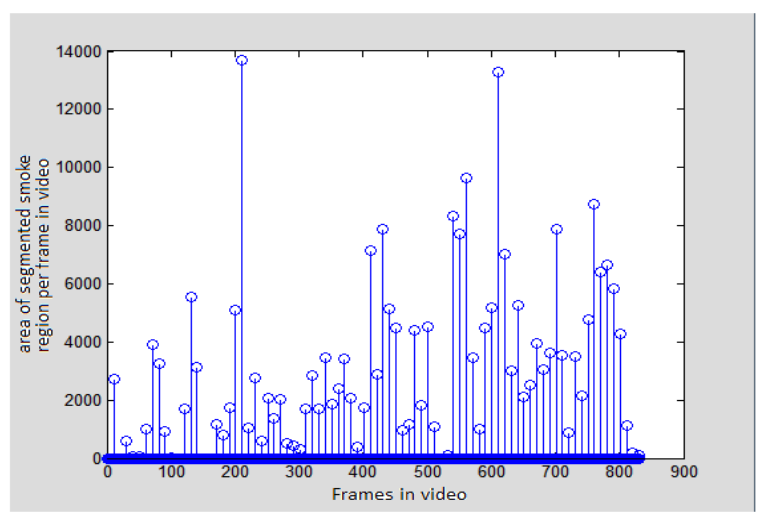

(c) 


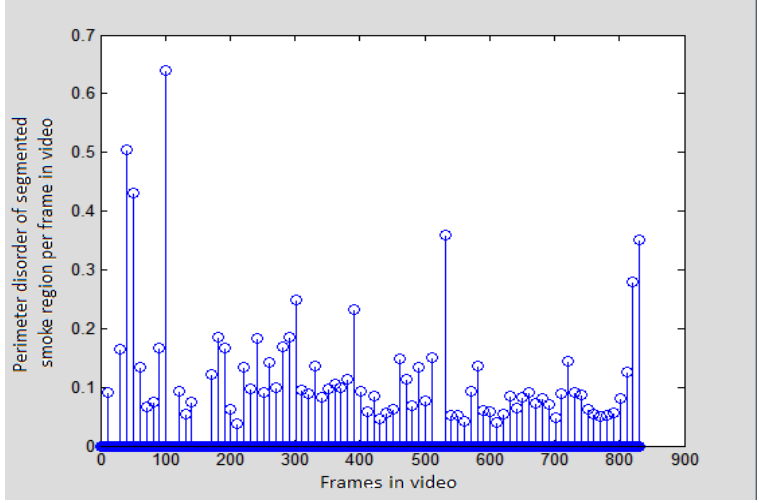

(d)

Fig 2: (a) a video having smoke, (b) segmented region of interest, (c) \& (d) area \& perimeter disorder resp. of segmented smoke per frame in corresponding video.

\subsection{Classification using SVM classifier}

SVM is a set of related supervised learning methods that analyze data and recognize patterns, thus it is employed in detection method for features based classification. To train the SVM, features of segmented smoke regions are computed and collected from video frames. With the help of these features, obtain the main parameters for SVM. Therefore, the segmented candidate smoke regions are further checked by the trained SVM classifier and the false regions can be avoided. Of course, static features can help filter the candidate regions segmented from video frames, but are not enough to describe the smoke changing in video sequences, so use along with it dynamic features to show segmented smoke region. As an example of this step results are shown in Fig 3:

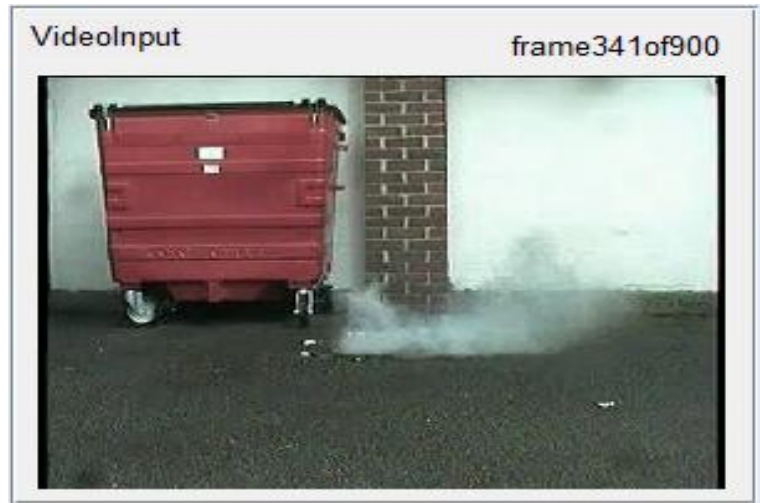

(a)

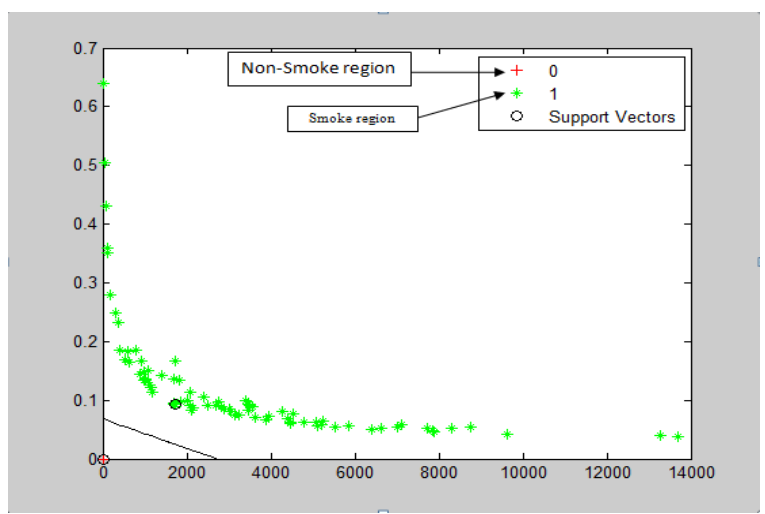

(b)

Fig 3: (a) a video having smoke (b) results of SVM.

\subsection{Generating alert message of smoke}

On the basis of result of classification phase i.e. whether the segmented region is smoke or non smoke an alert message of smoke presence in video is generated on screen [4].

\section{EXPERIMENTAL RESULTS}

The proposed method of smoke detection in video is implemented on Intel Core 2 Duo, $2.20 \mathrm{GHz}$ processor, with 4 GB RAM, Windows Vista OS, and using MATLAB software.

The summary of smoke detection test results in following video input is shown below:

\subsection{Video Input}

Fig 4: (a)-(d) shows video input at frame $=1,101,201,301$ as an example for result analysis.

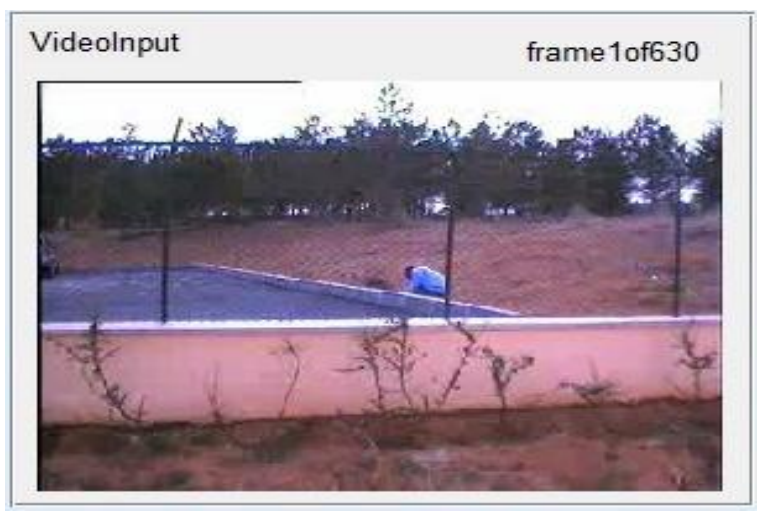

(a) At frame=1

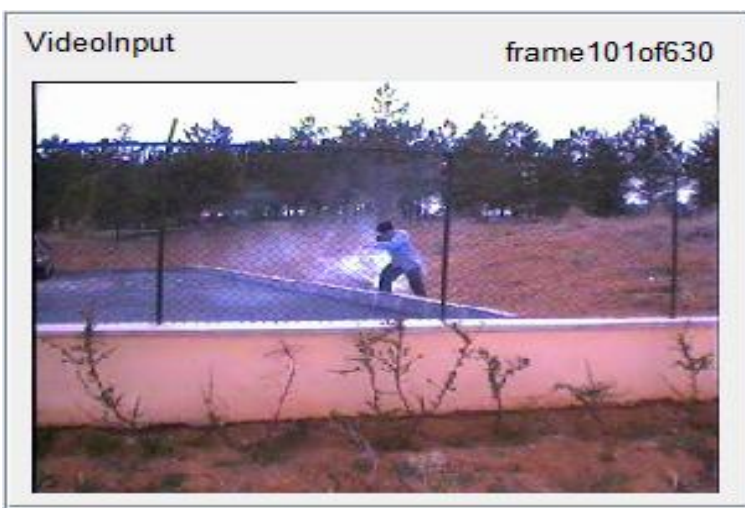

(b) At frame $=101$

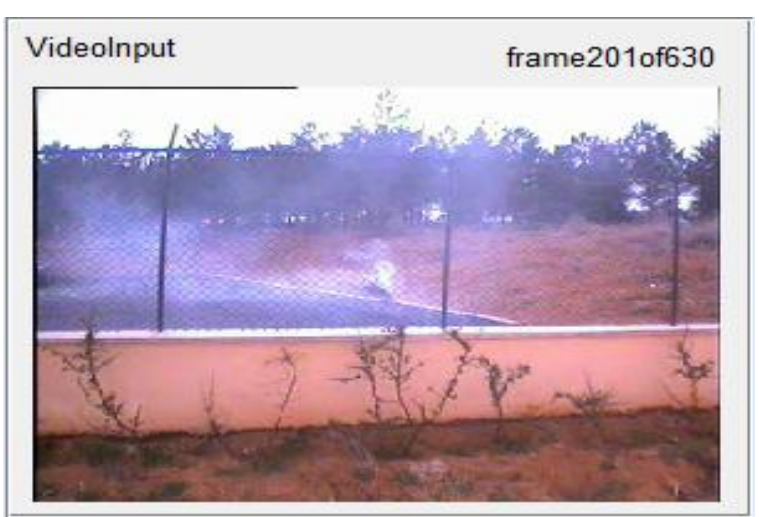

(c) At frame $=201$ 


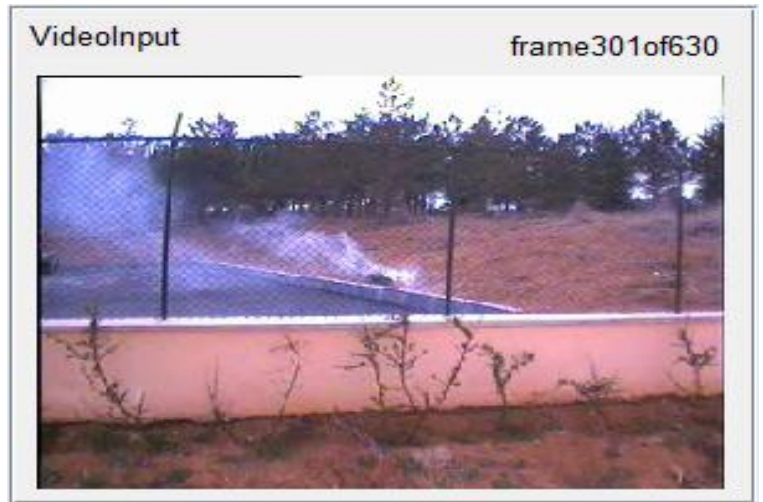

(d) At frame $=301$

Fig 4: Video input to smoke detection system

\subsection{Detection of moving region}

Fig 5: (a)-(d) shows moving region in video input in Fig 4, at frame $=1,101,201,301$.

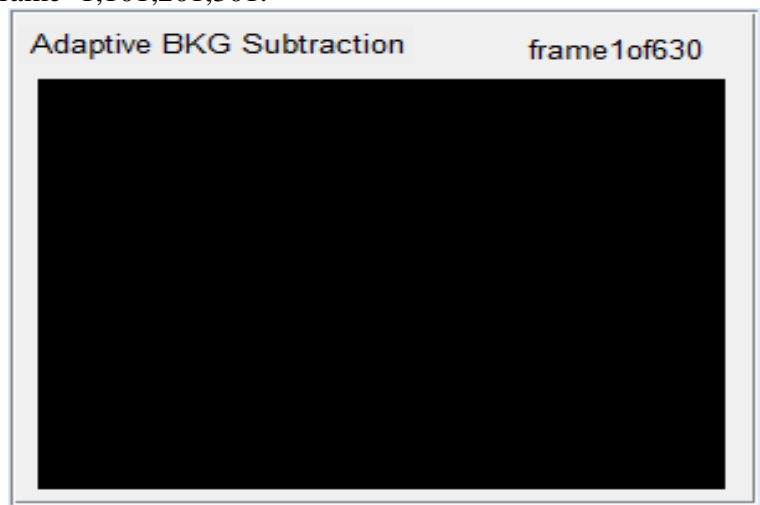

(a) At frame $=1$

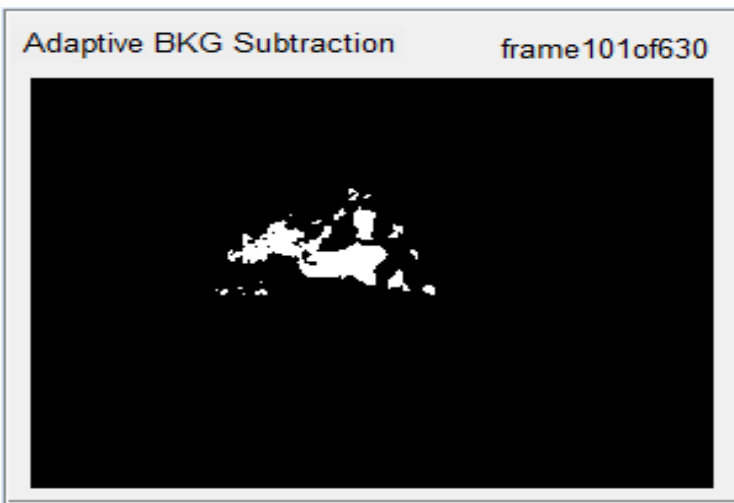

(b) At frame $=10$

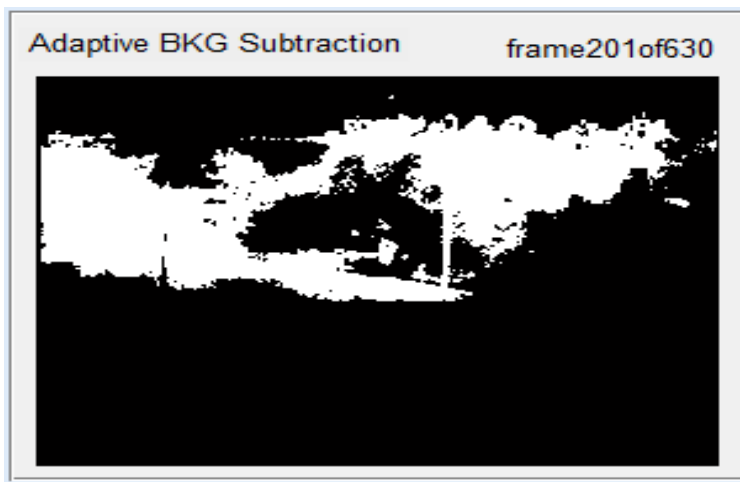

(c) At frame $=201$

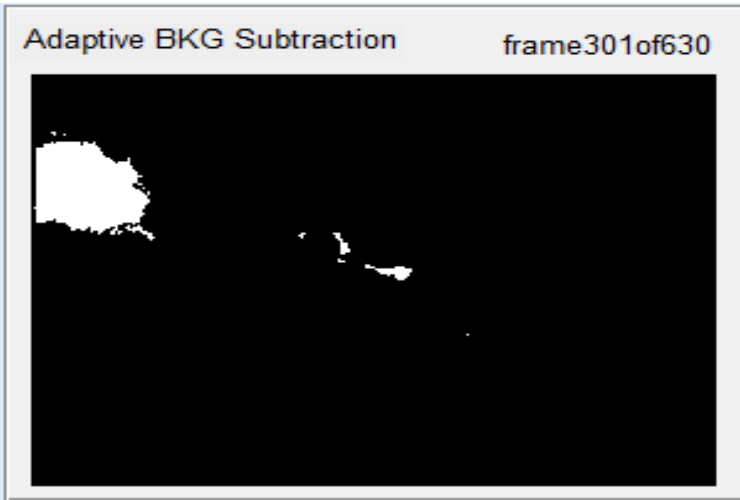

(d) At frame $=301$

Fig 5: Detection of moving region

\subsection{Detection of region having smoke color}

Fig 6: (a)-(d) shows region that match with smoke color in video input in Fig 4, at frame=1,101,201,301.

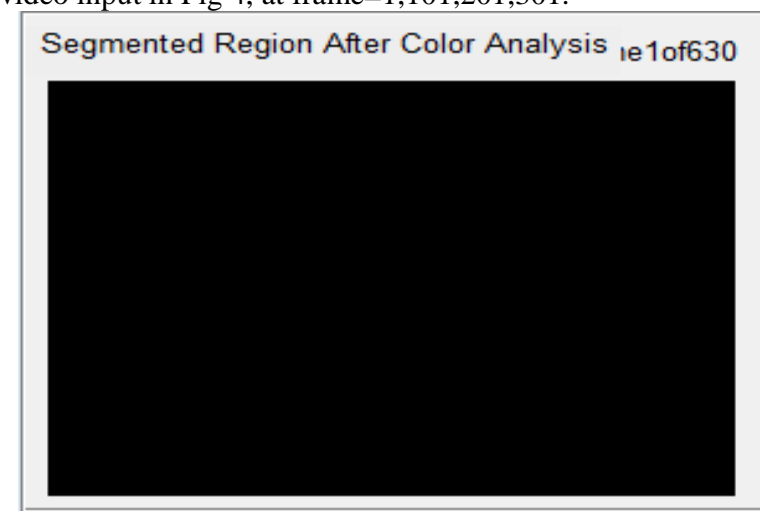

(a) At frame $=1$

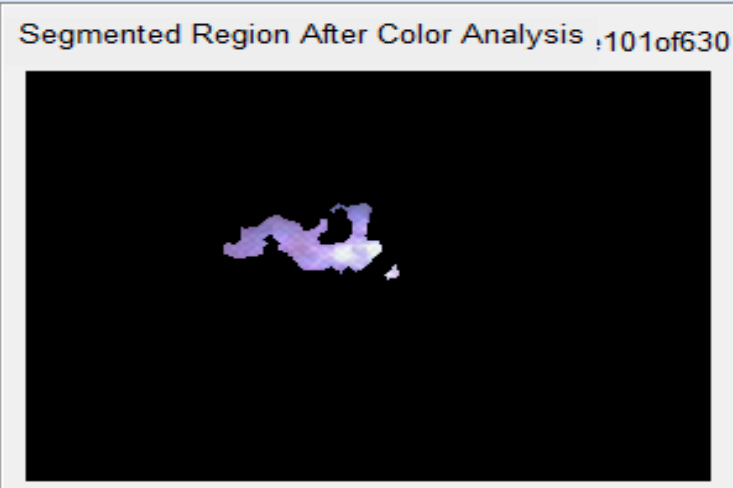

(b) At frame $=101$

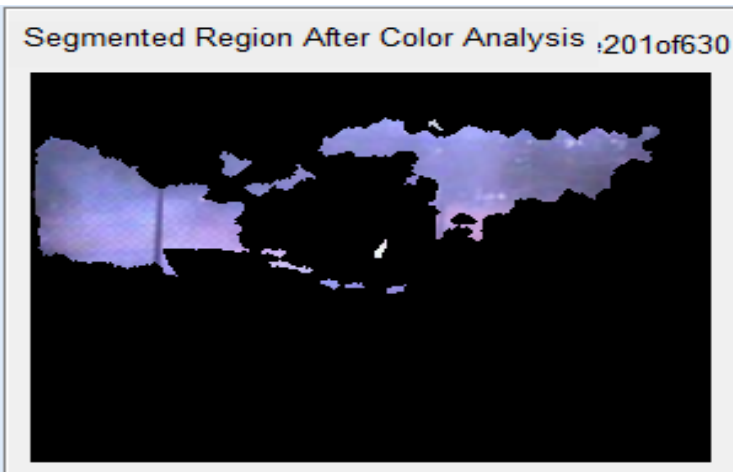

(c) At frame $=201$ 


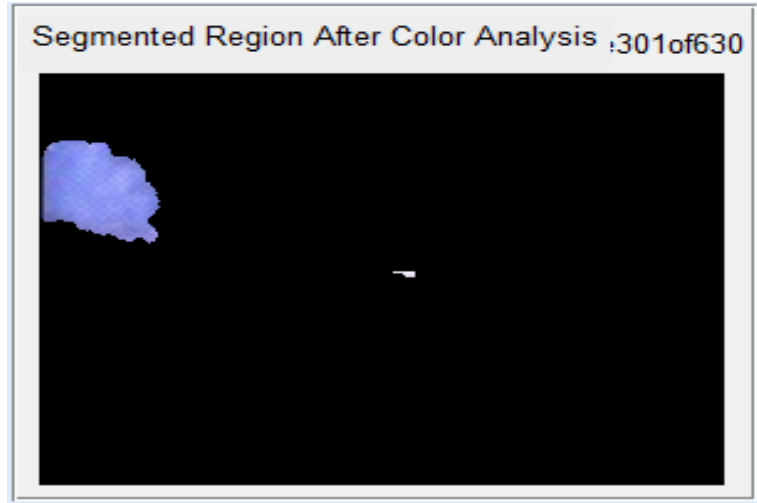

(d) At frame $=301$

Fig 6: Detection of region that match with smoke color

\subsection{Segmented smoke region}

Fig 7: (a)-(d) shows segmented smoke region in video at frame $=1,101,201,301$ for the input video in Fig 4 .

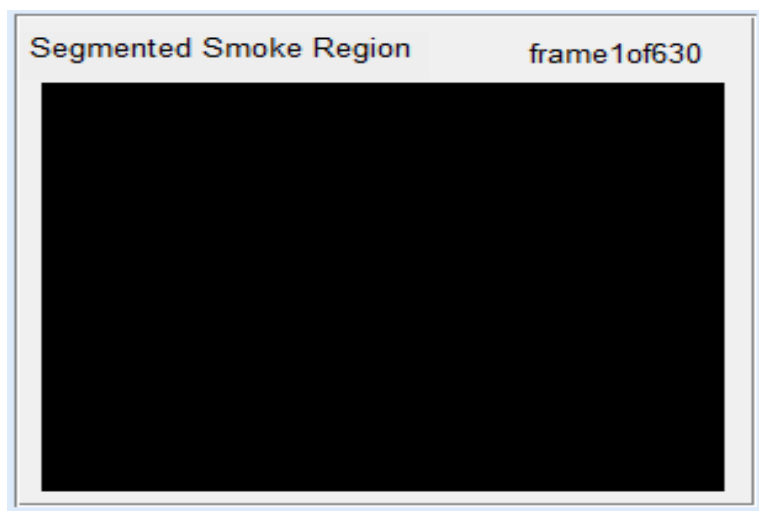

(a) At frame=1

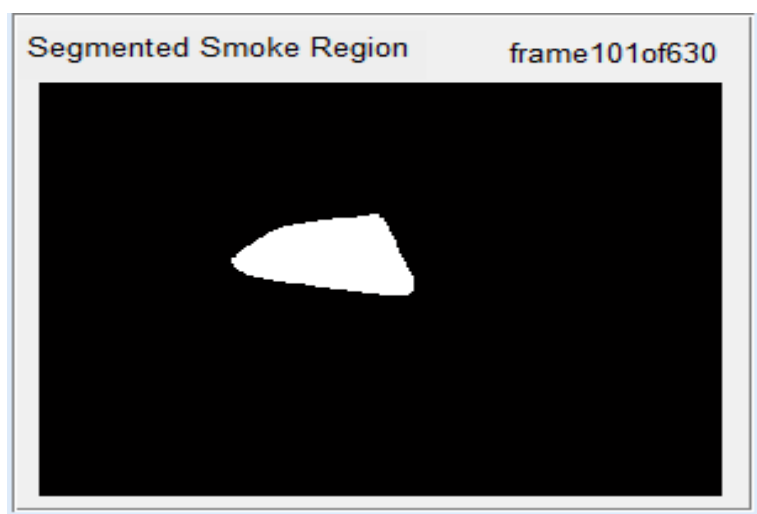

(b) At frame $=101$

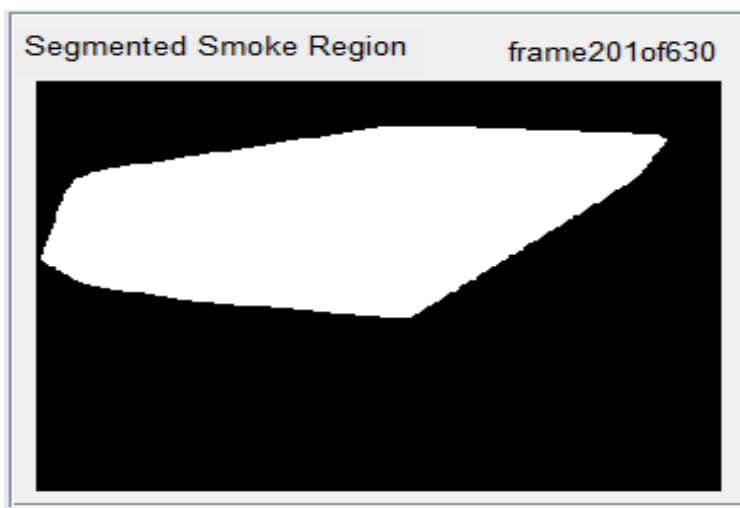

(c) At frame $=201$

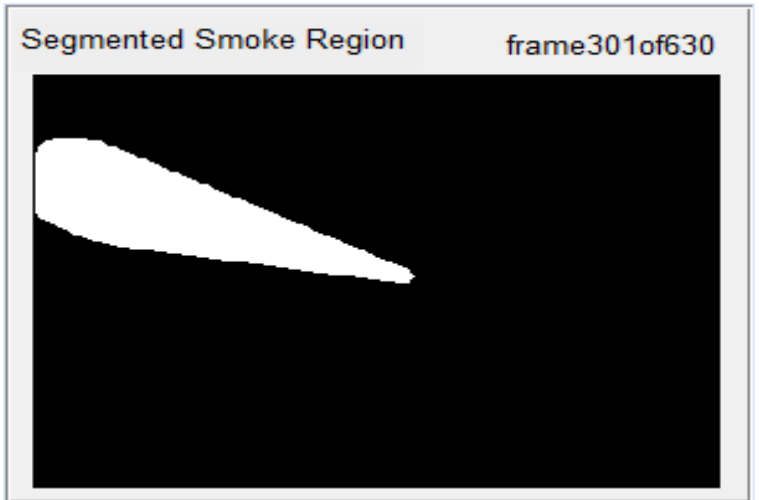

(d) At frame $=301$

Fig 7: Segmented smoke region

\subsection{Performance comparison}

To measure the performance of proposed method the true positive rate (TPR) i.e. correctly detected smoke, the false positive rate (FPR) i.e. recognizing a non-smoke region as a smoke region, and the missing rate (MR) i.e. not recognizing real smoke region are calculated [10]. The proposed approach achieved an average TPR of $96.84 \%$ and an average MR of $3.16 \%$; however an average FPR is $0 \%$. These results are improved as compared to methods in [9] [10]. The test results performed on 5 videos are shown in the Table 1 below:

The performance test results between proposed method and comparative methods [9] and [10] are shown in Fig 8:

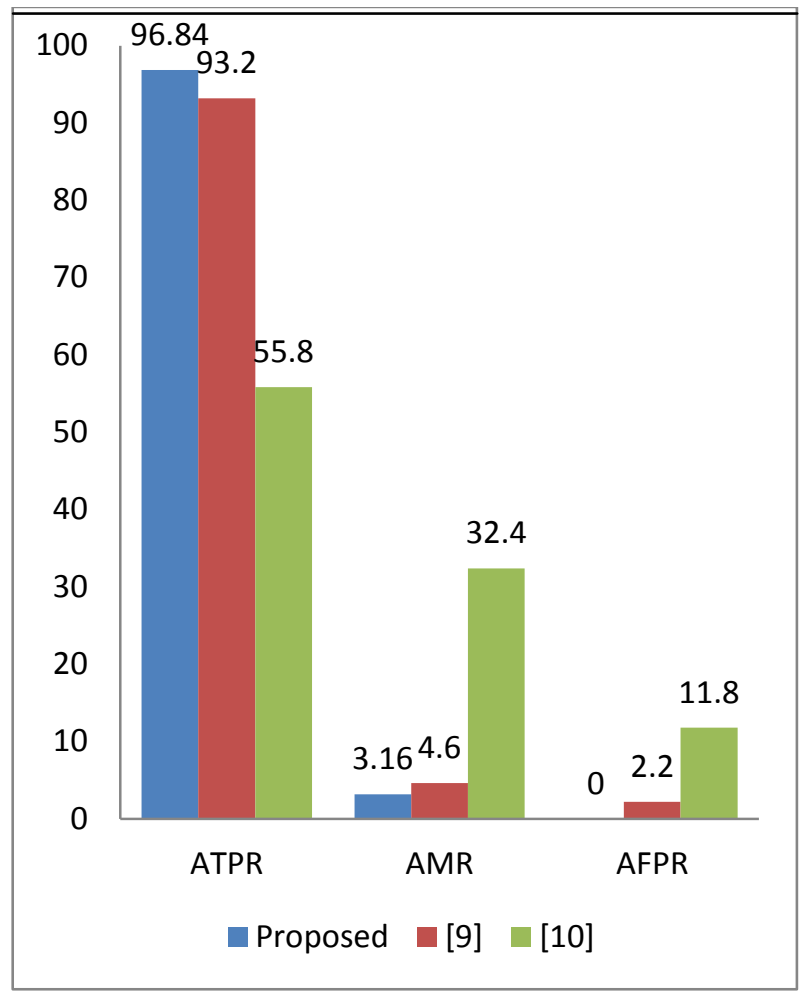

Fig 8: The performance test results between proposed method and comparative methods. 
Table 1. Smoke Detection results in 5 videos

\begin{tabular}{|l|c|c|c|c|c|c|c|c|}
\hline $\begin{array}{c}\text { video Input } \\
\text { (\# of frames } \\
\text { processed) }\end{array}$ & $\begin{array}{c}\text { \#Frame } \\
\text { having } \\
\text { smoke }\end{array}$ & $\begin{array}{c}\text { \# non } \\
\text { smoke } \\
\text { Frame }\end{array}$ & $\begin{array}{c}\text { \# Frame } \\
\text { smoke } \\
\text { detected }\end{array}$ & $\begin{array}{c}\text { \# Frame } \\
\text { no smoke } \\
\text { detected }\end{array}$ & $\begin{array}{c}\text { Smoke } \\
\text { Detected } \\
\text { (Yes/No) }\end{array}$ & TPR & MR & FPR \\
\hline sBehindtFence (62) & 58 & 4 & 57 & 5 & Yes & 98.27 & 1.72 & 0 \\
\hline sMoky(89) & 74 & 15 & 71 & 18 & Yes & 95.94 & 4.05 & 0 \\
\hline forest1(19) & 19 & 0 & 18 & 1 & Yes & 94.74 & 5.26 & 0 \\
\hline forest5(21) & 21 & 0 & 20 & 1 & Yes & 95.23 & 4.76 & 0 \\
\hline Viptraffic (12) & 0 & 12 & 0 & 12 & No & 100 & 0 & 0 \\
\hline Total:5 videos(203) & 172 & 31 & 166 & 37 & Result=Yes/No & ATPR=96.84 & AMR=3.16 & AFPR=0 \\
\hline
\end{tabular}

\section{CONCLUSION}

An efficient and responsive method for smoke detection in video is proposed. In first step, all the moving object in video are detected, which are in second step, checked for smoke color and object not having smoke color are removed and object with smoke color are checked for smoke in next step. In classification or decision step area and perimeter disorder characteristic of smoke region are used. Hence the combination of motion, color and other dynamic features like area, perimeter disorder provide more accurate results.

The performance of the proposed method is tested on 5 sets of videos containing both smoke and non-smoke video. The method achieves up to $96.84 \%$ correct smoke detection rate and result in a $3.16 \%$ and $0 \%$ missing and false detection rate respectively.

\section{ACKNOWLEDGMENTS}

Ashish Narwade thanks to Prof. V. A. Chakkarwar, Asst. Professor, Computer Science \& Engineering Department, Government College of Engineering, [Autonomous], Aurangabad, for her constant support and helping out with the preparation of this paper. Author thanks to all the faculty members of the Computer Science Department, Prof. V. P. Kshirsagar, HOD and Dr. P. S. Advani, Principal, Government College of Engineering, Aurangabad, for their constant support to our work.

\section{REFERENCES}

[1] S. Surit, W. Chatwiriya, "Forest Fire Smoke Detection in Video Based on Digital Image Processing Approach with Static and Dynamic Characteristic Analysis", in IEEE First ACIS/JNU ICC, Networks, Systems and Industrial Engineering, pp.35-39, 2011.

[2] A. A. Narwade, Prof. V. A. Chakkarwar, "Smoke Detection in Video for Early Warnings Using Static and Dynamic Features", in International Journal of Research in Engineering and Technology, Bangalore, pp.610-614, volume2, issue 11, Nov-2013.
[3] R. T. Collins, A. J. Lipton, T. Kanade, "A System for Video Surveillance and Monitoring", Proc. of American Nuclear Society 8th Int. Topical Meeting on Robotics and Remote Systems, Pittsburgh, PA, April 25-29, 1999.

[4] Tjokorda Agung, Budi W., Iping Supriana Suwardi, "Fire Alarm System Based-on Video Processing", in International Conference on Electrical Engineering and Informatics, Bandung, Indonesia, July 17-19, 2011.

[5] Turgay celik, Huseyin Ozkaramanli and Hasan Demirel, "Fire and Smoke Detection without Sensors: Image Processing Based Approach", in 15th European Signal Processing Conference (EUSIPCO 2007), Poznan, Polan, pp.1794-1798, September 3-7, 2007.

[6] Ali Rafiee, Reza Tavakoli, Reza Dianat, Sara Abbaspour, "Fire and Smoke Detection using Wavelet Analysis and Disorder Characteristics", in IEEE, pp.262-265, 2011.

[7] Vipin V, "Image Processing Base Forest Fire Detection", in IJETAE, Volume 2, Issue 2, pp. 87-94, February 2012.

[8] Yue Wang, Teck Wee Chua, Richard Chang and Nam Trung Pham, "Real-Time Smoke Detection Using Texture and Color Features", in 21st International Conference on Pattern Recognition (ICPR 2012), Tsukuba, Japan. pp. 1727-1730, November 11-15, 2012.

[9] B.U. Töreyin, Y. Dedeoglu and A. E. Cetin, "Contour based smoke detection in video using wavelets", in Proceeding 14th European Signal Processing Conference, EUSIPCO, 1-5, Sep. 4-8, 2006.

[10] JoonYoung Kwak, ByoungChul Ko, Jae-Yeal Nam, "Forest smoke detection using CCD camera and spatial temporal variation of smoke visual patterns", in 2011 Eighth International Conference Computer Graphics, Imaging and Visualization, Singapore, August $17-19$, 2011. 


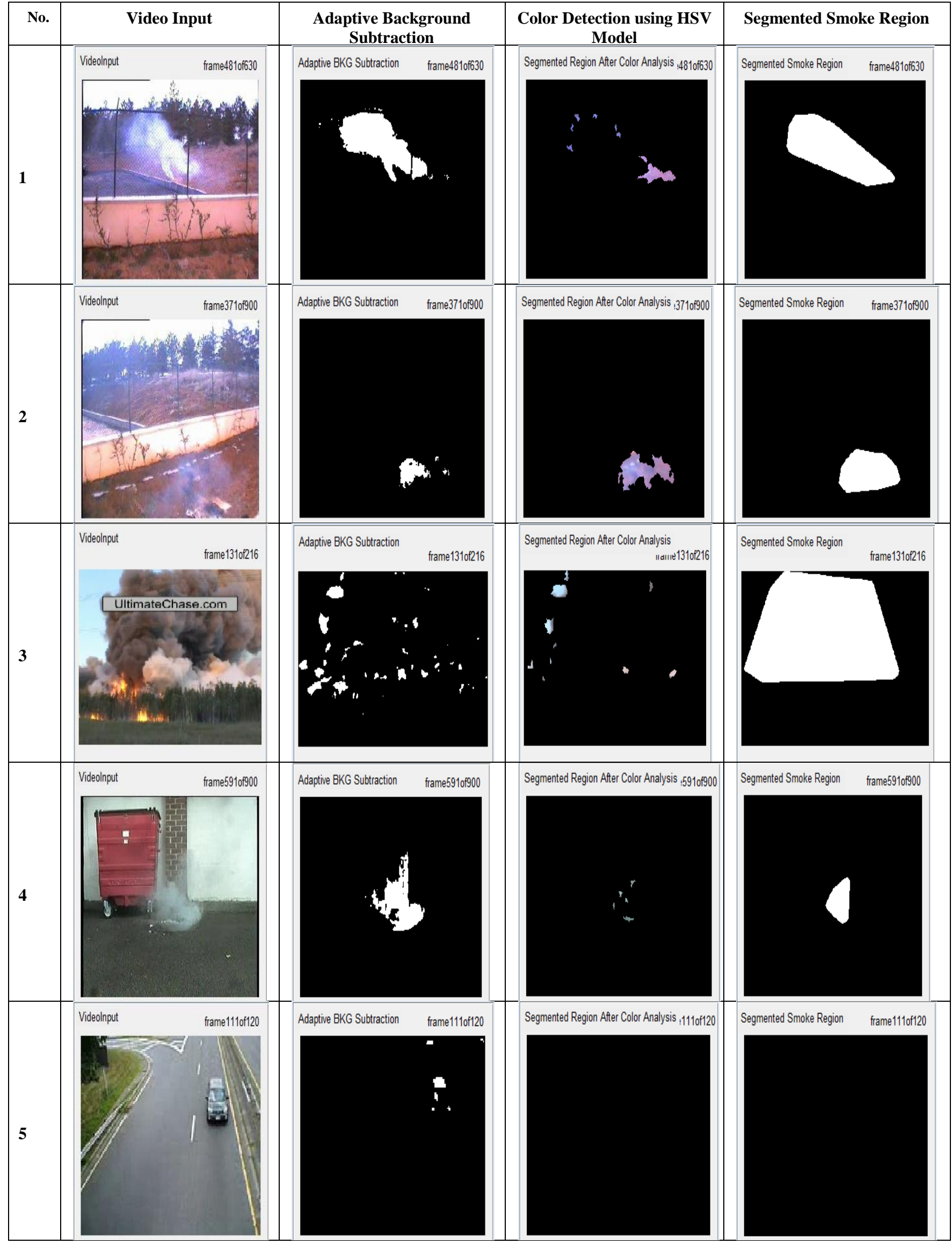

Table 2. Demonstration of Smoke Detection in 5 videos (1-4 with smoke and 5 with non-smoke video). 